\title{
Study of fungical conjunctival microbiota of students from the Health Sciences area of the Centro Universitário de Volta Redonda - UniFOA.
}

\author{
Aurilio Garcia ${ }^{1}$ \\ Jobson Bortot ${ }^{1}$ \\ Marco Antônio Ferraz ${ }^{1}$ \\ Lívia Mota ${ }^{1}$ \\ Tassius Borsatto ${ }^{1}$ \\ Antonio Neres Norberg ${ }^{2}$
}

Resumo

Os autores avaliaram o perfil da microbiota conjuntival fúngica de estudantes da área das Ciências da Saúde do Centro Universitário de Volta Redonda - UniFOA. Foram estudados 200 acadêmicos, sendo 100 pertencentes ao ciclo básico, com restrição ao ambiente hospitalar, e 100 do ciclo clínico, com efetivo contato. $\mathrm{O}$ material foi coletado do saco conjuntival e semeado em meio de cultura Sabouraud-dextrose-agar, e mantido em temperatura ambiente. As culturas foram positivas em 26 alunos, correspondendo a $13 \%$ do total examinado. Foram isolados os seguintes fungos: 7 (26,92\%) Candida albicans, 5 (19,23\%) Rhodotorula spp., 6 (23,07\%) Penicillium spp., 3 (11,53\%) Aspergillus niger, 3 (11,53\%) Fusarium spp., e 2 (7,69\%) Aspergillus fumigatus. Este resultado é compatível com a maioria dos estudos anteriores realizados por outros autores.

Palavras chave: microbiota conjuntival, ceratite fúngica, infecções oculares, acadêmicos de medicina

\begin{abstract}
The authors evaluated the profile of the fungical conjunctival microbiota of students from the Health Sciences area of the Centro Universitário de Volta Redonda - UniFOA. 200 academics had been studied, 100 of them belonging to the basic cycle of studies, with restriction to the hospital environment, and 100 of them from the clinical cycle, with effective contact. The material was collected of the conjunctival bag, sown in Sabouraud-dextrose-agar culture media, and kept in ambient temperature. The cultures had been positive in 26 academics, corresponding $13 \%$ of the examined total. The following fungi had
\end{abstract}


been isolated: 7 (26.92\%) Candida albicans, 5 (19.23\%) Rhodotorula spp., 6 (23.07\%) Penicillium spp., 3 (11.53\%) Aspergillus niger, 3 (11.53\%) Fusarium spp., and 2 (7.69\%) Aspergillus fumigatus. This result is compatible with the majority of the previous studies carried through by other authors.

Keywords: conjunctival microbiota, fungical keratitis, ocular infections, medical students

\section{Introdução}

Os fungos são microrganismos que podem ocasionar diversas patogenias em seres humanos. A gravidade das infecções fúngicas varia desde as formas cutâneas leves até quadros mais graves, como a septicemia, que poderá levar o paciente ao óbito. (Alecrim \& Teixeira, 1958; Höfling-Lima e col., 2005).

Várias espécies de fungos já foram isolados de seres humanos e responsabilizadas como agentes etiológicos de doenças. Desta maneira, Kurokawa e col. (1998) citaram que aproximadamente 300 espécies já foram identificadas como agentes etiológicos de infecções humanas. Os elementos fúngicos, de acordo com sua natureza, são classificados em filamentosos, leveduriformes e dimórficos.

Segundo considerações de Sidrim e col. (1999) e Höfling-Lima e col (2005), as infecções micóticas podem ocorrer e acometer todas as estruturas do olho. A primeira publicação sobre infecções micóticas oculares foi atribuída a Graefe (1854), com relato de micose do canal lacrimal (Rocha, 1952).

Sessenta espécies de fungos já foram isoladas do olho como causadoras de ceratites micóticas (Sidrim \& Rocha, 1999). O primeiro caso de micose ocular diagnosticado no Brasil e publicado como trabalho científico foi realizado por Silva (1921).

Diversos fatores induzem o início de um processo infeccioso micótico, seu prognóstico e sua evolução. As micoses oculares, incluindo as ceratites, manifestam-se clinicamente em situações de caráter oportunista e multifatorial (Wong e col., 1997), ocorrendo na presença de fatores que propiciam a modificação da microbiota conjuntival como o uso de medicações tópicas ou condições climáticas favoráveis, ou ainda pela destruição das defesas locais como trauma, cirurgias, doenças oculares prévias ou ainda pela diminuição da resistência do paciente em conseqüência de doenças sistêmicas ou uso de drogas imunossupressoras. O conhecimento da freqüência e dos fatores associados à doença micótica ocular pode ajudar na prevenção, no diagnóstico presuntivo e na conduta inicial nos casos de infecção ocular (Höfling-Lima e col., 2005).

Esse estudo teve como objetivo determinar o perfil da microbiota conjuntival fúngica em estudantes de Ciências da Saúde, usuários de lentes de contato, e orientá-los sobre os riscos de infecção em relação ao uso inadequado das referidas lentes. 


\section{Material e métodos}

Foram estudados 100 acadêmicos na área das Ciências da Saúde pertencentes ao ciclo básico, com restrição ao ambiente hospitalar e 100 do ciclo clínico, com efetivo contato. O material foi coletado no saco conjuntival com o auxílio de uma zaragatoa embebida em solução de salina estéril. Semeou-se em meio de cultura de Sabouraud-dextrose-agar em placas, que foram vedadas com fita adesiva e mantidas em temperatura ambiente. A identificação dos fungos filamentosos foi realizada pela observação dos caracteres culturais e os leveduriformes por provas bioquímicas, pelo sistema BioMerieux-Vitek.

\section{Resultados}

As culturas fúngicas foram positivas em 26 alunos, correspondendo a 13\% do total examinado. Foram isolados os seguintes fungos: 7 (26,92\%) Candida albicans, 5 (19,23\%) Rhodotorula spp., 6 (23,07\%) Penicillium spp., 3 (11,53\%) Aspergillus niger, 3 (11,53\%) Fusarium spp., e 2 (7,69\%) Aspergillus fumigatus (Gráfico 1).

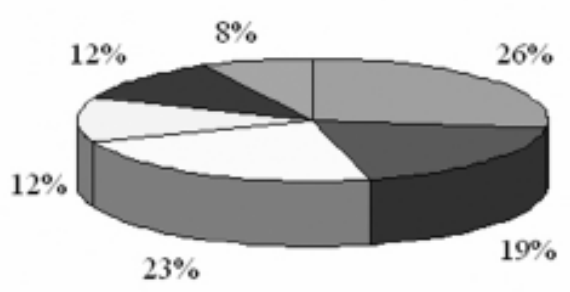

Gráfico 1: Culturas Positivas $\square$ Candida albicans

Rhodotonda spp

$\square$ Penicilfum spp

$\square$ Aspergillus niger

- Fusarim spp

Aspergillus funigatus

\section{Discussão}

Infecções oculares de etiologia fúngica podem ser consideradas como um sério problema oftalmológico em várias partes do mundo. Em algumas ocasiões pode evoluir para uma infecção fulminante e em alguns pacientes pode conduzir à cegueira. Oliveira e col. (2001) mencionaram que a prevalência das referidas micoses oculares é mais comum em regiões de clima quente, e geralmente está associada com traumatismo, doença corneana pré-existente, uso de esteróides e antibióticos ou lentes de contato. Esses autores consideraram ainda que apesar do progresso no diagnóstico e tratamento, a ceratite fúngica continua sendo um desafio para os oftalmologistas.

A contaminação das estruturas oculares pode ocorrer com muita facilidade por elementos fúngicos presentes no ambiente. Dessa maneira, os pesquisadores Tkachyk \& Khan (1977) estudaram a contaminação fúngica do ambiente hospitalar, e identificaram 44 
gêneros de fungos, entre eles espécies do gênero Penicillium e Candida albicans, fungos esses também encontrados nos estudos realizados por nossa equipe no material conjuntival de usuários de lente de contato.

Com relação à biota conjuntival, Vieira e col. (1999) e Wilson e col. (1969) relataram que elementos fúngicos são isolados do saco conjuntival de olhos saudáveis em uma incidência que varia de 2,9\% a 72\%, dependendo da atividade profissional do indivíduo. Trabalhos realizados por esses autores revelaram que a contaminação fúngica da mucosa conjuntival de cortadores de cana correspondeu a uma variação entre $38,5 \%$ a $72 \%$. Entre os fungos isolados nesses trabalhadores, com maior freqüência estão Aspergillus spp., Candida spp. E Penicillium spp.. Esses resultados corroboram nossas pesquisas, porém a incidência encontrada entre estudantes da área das Ciências da Saúde foi muito inferior, provavelmente pelo menor contato com o ambiente contaminado.

Como os estudantes objeto da nossa pesquisa não apresentavam sinais clínicos de complicações oculares, conclui-se que os fungos isolados eram constituintes da microbiota normal da mucosa conjuntival. Porém, deve-se considerar que o uso inadequado das lentes de contato, associado à presença de elementos fúngicos, poderá constituir um fator predisponente de infecções oculares.

\section{Bibliografia}

1. ALECRIM, E. \& TEIXEIRA, H. Fungos anemofílicos da cidade do Recife, Pernambuco, Brasil. An. Fac. Med. Univ. Fed. Pernamb., 18: 269-274, 1958

2. HÖFLING-LIMA, A. L.; FORSETO, A.; DUPRAT, J. P.; ANDRADE, A.; SOUZA, L. B.; GODOY, P. \& FREITAS, D. Estudo laboratorial das micoses oculares e fatores associados às ceratites. Arq. Bras. Ofmalmol., 68 (1): 21-27. 2005

3. KUROKAWA, C. S., SUGIZAKI, M. F. \& PEÇAROLI, M. T. S. Virulencia factors in fungi of systemic mycosis. Rev. Inst. Med. Trop. São Paulo, 40 (3): 125-135, 1998

4. OLIVEIRA, P. R.; RESENDE, S. M.; OLIVEIRA, F. C. \& OLIVEIRA, A. C. Ceratite fúngica. Arq. Bras. Oftalmol., 64: 75-79, 2001

5. ROCHA, M. Micoses em oftalmologia. Arq. Inst. Penido Burnier, 9 (1): 28-69, 1952

6. SIDRIM, J. J. C. \& ROCHA, M. F. G. Biologia dos fungos. In: Sidrim, J. J. C; Moreira, J. C. B. editores. Fumdamentos clínicos e laboratoriais da micologia médica, Ed. Guanabara Koogan, Rio de Janeiro, p.20-29, 1999

7. SILVA, L. Sobre um caso de úlcera ectimatóide pré-lacrimal esporotricósica com linfangite gomosa da face. Rev. Bras. Med., 35:115-118, 1921 
8. TKACHYK, S. J. \& KHAN, R. S. Airbourne mold survey, J. Asthma Res., 14: 103106, 1977

9. VIEIRA, L. A.; BELFORT, R.; FISCHMAN, O. F. \& SCARPI, M. Estudo da flora fúngica da conjuntiva normal de cortadores de cana na região canavieira de Santa Rita, Paraíba, Brasil. Arq. Bras. Oftalmol., 52: 63-67, 1989

10. WILSON, L. A.; AHEARN, J. G.; JONES, D. B. \& SEXTON, R. R. Fungi from the normal outer eye. Am. J. Ophtalmol., 67: 52-56, 1969

\section{Informações bibliográficas:}

Conforme a NBR 6023:2002 da Associação Brasileira de Normas Técnicas (ABNT), este texto científico publicado em periódico eletrônico deve ser citado da seguinte forma: GARCIA, A.; BORTOT FILHO, J; BAPTISTA, M. A. F. B.; MOTA, L. O. D.; SACCOMANI, T. B.; NORBERG, A. N. Study of fungical conjunctival microbiota of students from the Health Sciences area of the Centro Universitário de Volta Redonda UniFOA.. Cadernos UniFOA, Volta Redonda, ano 2, nº 3, mar. 2007. Disponível em: $<$ http://www.unifoa.edu.br/pesquisa/caderno/edicao/03/129.pdf > 